\title{
EVALUATION OF USING MINERALIZED PLASMATIC MATRIX IN OSSEOUS REGENERATION (EXPERIMENTAL AND HISTOMORPHOMETRIC STUDY ON RABBITS)
}

\author{
Marwa A. Ghanem ${ }^{1} B D S$, Ahmed A. Sharara ${ }^{2} P h D$, Ahmed O. Sweedan ${ }^{3} P h D$, \\ Nesma M. Khalil ${ }^{4} P h D$
}

\begin{abstract}
INTRODUCTION: The use of platelet concentrates is recent and its efficiency remains controversial. The mineralized plasmatic matrix (MPM), is the latest innovation of platelet concentrates (i.e. PRP and PRF). A new concept of fabricating growth factors-enriched bone graft matrix has been introduced since 2010. It utilizes altering the centrifugation speed and time to produce much larger, denser and richer fibrin matrix containing growth factors, known as Sticky bone. MPM combines the best of both worlds; its mineral phase qualifies it as an osteoconductive scaffold for bone formation while its platelet concentrate phase grants it an osteoinductive property by the slow release of growth factors.

OBJECTIVES: To evaluate the effectiveness of using MPM, compared to the use of bone graft alone, to assess the exact effect of each in enhancing the osteogenic differentiation and bone formation.

MATERIALS AND METHODS: Ten healthy adult white Albino New Zealand rabbits, after creating two osseous defects in the right femur in each. The defects (upper and lower) were divided in to two groups according to the material used for filling: Group A (Control): in the upper defect, bone graft only was added in the lateral side of the right femur.

Group B (Study): in the lower defect, MPM was addedin the lateral side of the right femur.

After 2 \&8 weeks respectively postopertively,sacrification of the rabbits was done.

RESULTS: Data collected from histologicalresults revealed that MPM enhanced bone formation where increased amount of new bone formation was observed in the study group in relation to the control group. At the end of the experimental period,the defect area was almost filled with mature bone which occupied a greater surface area in the study group. Histomorphometric analysis revealed that there was increase in the mean percentage of bone surface area in the study group in comparison to the control group. This increase was statistically significant in the 8 weeks period.

CONCLUSIONS: MPM proved its effeciency in enhancement of osseous regeneration.

KEYWORDS: Platelet rich fibrin (PRF), MPM (Mineralized plasmatic matrix), Platelet rich plasma (PRP), bone graft, osseous regeneration.

1- BDS, MS, Faculty of Dentistry, Alexandria University, Egypt.

2- Professor of Oral and Maxillofacial Surgery, Department of Oral and Maxillofacial Surgery Faculty of Dentistry, Alexandria University, Egypt.

3- lecturer of Oral and Maxillofacial Surgery, Department of Oral and Maxillofacial Surgery Faculty of Dentistry, Alexandria University, Egypt.

4- lecturer of Oral Biology, Department of Oral Biology, Faculty of Dentistry, Alexandria University, Egypt.
\end{abstract}

Corresponding author:

E-mail: morodentist_1987@hotmail.com

\section{INTRODUCTION}

The repair and regeneration of bone is a major issue in the Oral and Maxillofacial field and for the whole human body in general. Bone loss is caused by many diseases (congenital or degenerative), traumas and surgical procedures; it is a problem for functionality and is having an ever-increasing social impact (1).

Bone grafting is often necessary to provide support, fill voids and enhance biologic repair of skeletal defects (2). It includes autografts, allografts, xenografts and alloplastic materials $(3,4)$.

Autogenous bone is an ideal bone graft having osteogenic, osteoconductive and osteoinductive potential. Donor site morbidity such as neurovascular injury, infection, haematoma formation and chronic pain are well known complications of autogenous bone graft as well as it is limited in quantity (5). Therefore; efforts have focused on finding ideal materials that could be used as substitutes for autografts (6). The selection of the most appropriate scaffolds is an important step towards the regeneration of hard tissue (7).

Platelet-derived Growth Factors are biologically active peptides that enhance tissue repair mechanisms, cell proliferation and regulate angiogenesis (8).

Platelets are easily collected from the blood stream and are concentrated in a small volume of plasma known as platelet-rich plasma (PRP) (9). It is the first form of autologous platelet concentrate to replace commercial fibrin glue. The preparation of PRP involves the isolation of PRP after which gel formation is accelerated using calcium chloride and bovine thrombin. It has been discovered that the use of bovine thrombin may be associated with the development of antibodies to the factors $\mathrm{V}, \mathrm{XI}$ and thrombin, resulting in the risk of life coagulopathies. Bovine thrombin preparations have shown to contain factor V, which could result in the stimulation of the immune system when challenged with a foreign protein (10).

Platelet rich fibrin (PRF)was first described by Choukroun etal (11). This second-generation platelet 
concentrate eliminated the risks associated with the use of bovine thrombin (12). The natural concentrate of PRF is produced without any anticoagulants or gelifying agents (13). It is an autologous fibrin matrix containing many kinds of growth factors and cytokines (14). Growth factors are known to promote cell proliferation, cell differentiation, cell motility, and matrix synthesis by binding to specific cellsurface receptors (15). It is generally accepted that growth factors have an essential role in the healing process and tissue formation $(16,17)$.

Platelet rich fibrin has several advantages over traditionally prepared platelet-rich plasma including ease of preparation and lack of biochemical handling of blood which makes this preparation strictly autologous (18).

It can be used in conjunction with bone grafts, which offers several advantages including promoting wound healing, bone growth and maturation, graft stabilization, wound sealing and hemostasis, and improving the handling properties of graft materials (19).

The latest innovation of platelets concentrates (i.e PRP and PRF) is the mineralized plasmatic matrix. Therefore, it has been suggested that upon further optimization, MPM may serve as a third generation of platelet concentrate with potential application in various fields. The MPM is a modification of and PRF initially presented by Perisse (20) in 2012 and modified by ElMoheb (21) in 2014.

The advantage of the MPM is the integration of bone grafts particles inside the fibrin network that is not present in old autologous growth factors membranes in PRF or PRP. In fact, the bone grafting materials are prepared and mixed with the autologous growth factors to produce the MPM or what is called Sticky bone. This offers the MPM the positional stability by stabilizing the bone particles, preserving its shape with subsequent "in situ" immobilization of the component of ridge preservation materials (22).

In this study, we introduced an easy technique utilizing (MPM:mineralized plasmatic matrix) for reconstruction of bony defects and evaluation ofits effectiveness in osseous regeneration.

\section{MATERIALS AND METHODS}

\section{Materials}

\section{A) Experimental animals}

The study was performed after obtaining the approval of the Research Ethics Committee, Faculty of Dentistry, Alexandria University.

The study that was conducted on 10 healthy adult white New Zealand rabbits (age 2-2.5 months and weighing 4-5 kgs approximately), the defects were divided in to two groups according to the material used for filling:

Group A (Control): in the upper defect, Bone graft only was added in the lateral side of the right femur.

Group B (Study): in the lower defect, MPM was added in the lateral side of the right femur.

\section{B) Bone graft: Hypro-Oss ${ }^{\circledR}$}

Hypro-Oss ${ }^{\circledR}$ (Hypros.r.o, Germany,Europe) is a natural bovine bone substitution material for permanent filling and reconstruction of bone defects. Each granule consists of 30\% Atelo-Collagen Type I and 70\% hydroxyapatite. Hypro-Oss ${ }^{\circledR}$ is produced by using patented atelopeptidation and lyophilization processes.

Hypro-Oss ${ }^{\circledR}$ is a sterile medical device class III, ISO and CE-certified.

\section{Methods}

The experimental procedure was held in the animal house at Faculty of Agriculture, Alexandria University. Animals were kept under safe conditions during the time of the study.Rabbits were kept under the same nutritional and environmental conditions throughout the whole period of the study. Each animal received a dose of antibiotics in the form of Ampicillin (Epicocillin, EPICO, Cairo, Egypt) 25 $\mathrm{mg} / \mathrm{kg}$ body weight by intra-muscular injection just before the operation.

\section{Operative phase}

\section{Preparation of MPM}

- 5 CC of rabbits venous blood was taken from the marginal ear vein, and the blood was added to the non-coated plain vacutainers. The blood in the test tubes was centrifuged at 2400-2700rpm for 2-4 minutes (20,21). The non-coated tube showed 2 different layers:

The upper layer showed a liquid yellow plasma while the lower layer showed red blood cells layer which was discarded (Figure 1a), while the yellow part was then collected using a syringe, and added to cup that contains the bone graft material. (Figure 1b)

The whole thing was mixed for few minutes to polymerize in order to obtain the MPM (sticky bone), which is yellowish in color. (Figure 2a)

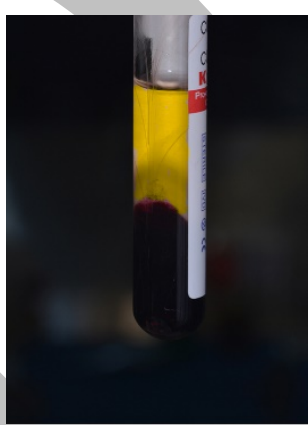

(A)

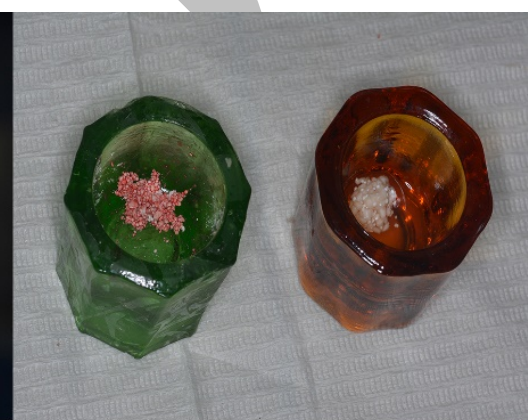

(B)
Figure 1: (A) Showing liquid yellow plasma layer on the top of the red blood cell layer after test tube centrifugation.

(B) Showing two cups, the right one contains MPM while the left one contain bone graft alone.

\section{Anaesthesia}

All operating procedures were performed under general anaesthesia. Each animal was generally anaesthetized via intramuscular injection of Xylazine HCL 2\% (Xyaline) (Bayer Inc, Toronto,ON,Canada), the dose of which was calculated on basis of $2 \mathrm{mg} / \mathrm{kg}$ body weight and intramuscular injection of ketamine HCL 10\% (Ketamine) (Alfas an Inc.,Utrecht,Holland) in a dose of $3 \mathrm{mg} / \mathrm{kg}$ body weight.

\section{Surgical operation}

The femur area wasshaved and sterilized with bovidone Iodine (Betadine) (Nile Company, Cairo, Egypt). About $5 \mathrm{~cm}$ incision was done. Once the skin was incised, the fascia was cut with a pair of scissors until the bone was reached. The periosteum was reflected with a periosteal elevator. Two surgically created bone defects of $5 \mathrm{~mm}$ diameter and $5 \mathrm{~mm}$ depth, and $5 \mathrm{~cm}$ apart from each other were prepared in the right side by the aid of trephine bur (Tut Company, Cairo, Egypt).

The defect was filled with different materials according to the study group (Figure $2 b$ ): 


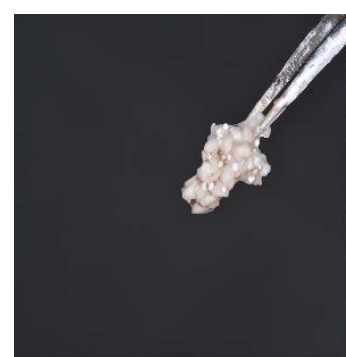

(A)

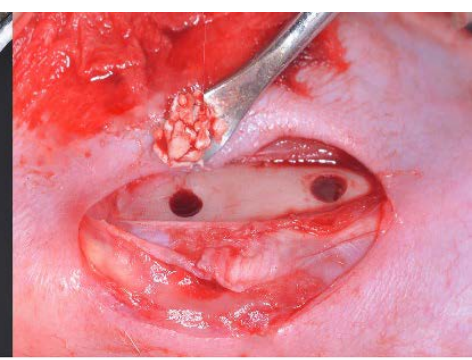

(B)
Figure 2: (A) Showing MPM (sticky bone)

(B) Showing the two defects ready to be filled with different materials.

Group A (Control): In the upper defect, Bone graft only was added in the lateral side of the right femur.

Group B (Study): In the lower defect, MPM was added in the lateral side of the right femur.

The flaps were advanced to their original position using tension free wound closure and sutured using simple interrupted sutures byusing polyglycolicpolylactic 3-0 suture material (Vicryl, Ethicon, Cornelia, GA, USA) for deep layers and 4-0 polypropylene suture material (Prolene, Ethicon, Cornelia, GA, USA).

\section{Postoperative phase}

\section{Clinical follow up period}

1-All rabbits received antibioticsin the form of Ampicillin (Epicocillin) (Eipico,Egypt) 1gm IM immediately postoperatively.Then they also received a dose of Amoxycillin (amoxil)(,gsk,Cairo,Egypt) $250 \mathrm{mg}$ IM for 3 days postoperatively.

2-All rabbits received the same course of anti-inflammatory and analgesic in the form of diclofenac potassium (Cataflam)(Novartis,Switzerland) 25mg/day IM for 3 days.

3-Animals were kept under observation to assess the presence or absence of any infection, wound dehiscence or graft rejection.

4-Animals were kept on soft diet in the firstweek postoperatively.

\section{Animal Sacrification}

Rabbits were sacrificed at (2 - 8 weeks), (5 rabbits each time), respectively postoperatively by giving them an overdose of anaesthesia pentobarbital Sodium (Nembutal) (Akron pharmaceuticals, US).

All specimens were labeled and fixed in $10 \%$ neutral buffered formalin for one day, after which segments containing the surgical sites were dissected out and left in the same fixation for 5 days.

\section{Histological procedure}

After fixation, specimens were decalcified in $8 \%$ Trichloroacetic acid solution, washed, dehydrated in ascending concentrations of ethanol, cleared with xylene, infiltrated and embedded in paraffin wax.

Serial thin sections of $5 \mu \mathrm{m}$ thickness were cut using a rotary microtome. Sections were stained with Haematoxylin and Eosin stains (H\&E) (23) to evaluate quality of the newly formed bone.

\section{Histomorphometric analysis}

Computer-assisted histomorphometry (24) was performed in order to compare the percentage of the surface area of the newly formed bone in different groups.

Histomorphometrical evaluation of the percentage of the bone surface area

Morphometric evaluation of the percentage of surface area of the alveolar bone was assessed for each specimen using the (Image J 1.46) (25) software program.

\section{Steps of measuring percentage of bone surface area}

- Three sections of tissue from different standardized depths were used for quantification from each sample each separated from the other by about 100 microns.

- One photograph was taken from each section using the same magnification power.

- Three rectangles with standardized dimensions were drawn on a specific areas of the defect, which are,the upper left side,the lower left side and the middle right side.

- The total surface area of each rectangle was measured using the image $\mathrm{J}$ program by choosing the region of interest (ROI) manager, from tools from analyze and the measurement was recorded.

- The surface areas occupied by the marrow space were selected using the wand tracing tool and their total measurements were recorded.

- The two recorded measurements were subtracted to obtain the surface area occupied only by bone.

- The mean bone surface area of the three rectangles was calculated for each section.

- The mean of the values from the three sections was obtained and its percentage to the total area selected was calculated.

- The same procedure was repeated for each of the five animals in each group.

- The collected data of the percentage of bone surface area were statistically analyzed.

\section{Statistical analysis of the data}

Measurements for the percentage of bone surface area were collected from the images.Data were fed to the computer and analyzed using IBM SPSS softwarepackageversion 20.0. (Armonk, NY: IBM Corp) (26). Qualitative data were described using number and percent. The KolmogorovSmirnov test was used to verify the normality of distribution. Quantitative data were described using range (minimum and maximum), mean, standard deviation and median. Significance of the obtained results was judged at the $5 \%$ level, $(\mathrm{P} \leq 0.05)$.

\section{The used tests were}

\section{1- Student t-test}

For normally distributed quantitative variables, to compare between two studied groups.

\section{2- Mann Whitney test}

For abnormally distributed quantitative variables, to compare between two studied groups

\section{RESULTS}

\section{Clinical observations}

All rabbits survived through the entire study period. No significant complications or clinical signs of infection or wound dehiscence that would impair osseous regeneration throughout the whole follow up period. 


\section{Histological results}

H\&E stain was used to distinguish between immature woven bone and mature lamellar bone.

Light microscopic examination of the 2 groups to observe different levels of bone formation as well as the collagen content of the newly formed bone.

\section{Two weeks interval}

Light microscope examination of specimens taken from the defect site of group A (control) revealed limited amount of new bone formation extending from the margins of the defect. This newly formed bone consists of spicules containing haphazardly arranged osteocytes. The remaining part of defect contains fibrous connective tissue and remnants of the graft material. Cartilage like tissue could be also seen (Figure 3).

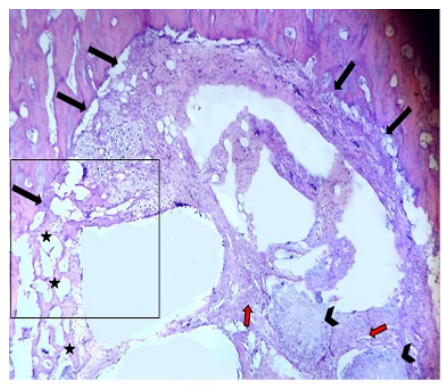

(A)

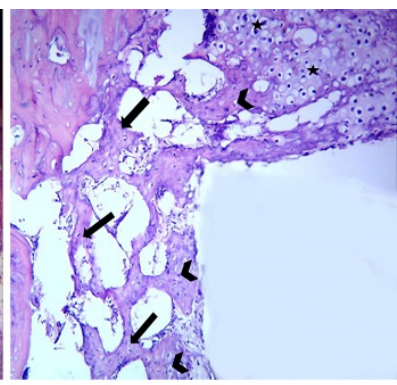

(B)
Figure 3: (A) LM of the defect site of control group (2 weeks) showing the newly formed bone (star) extending from the boundary of the defect (long black arrows). Other parts of the defect contain fibrous tissue (short red arrows) enclosing remenants of the graft material (arrow heads). (H\&E x 40).

(B) LM of higher magnification of the previous micrograph inset showing the newly formed bone spicules (arrows) containing numerous irregularly distributed osteocytes (arrow heads). Other parts of defect contain cartilage like tissue (stars). (H\&E x 100)

On the other hand, in group B (study), more numerous bony spicules were seen originating from all around the periphery of the bone defect. This newly formed bone contained numerous, large, irregularly arranged osteocytes. Osteoblasts cells were seen lining the endosteal surface of the bony spicules (Figure 4).

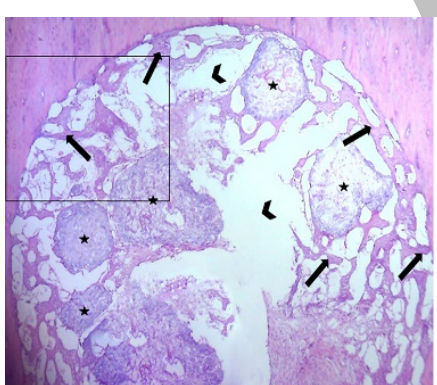

(A)

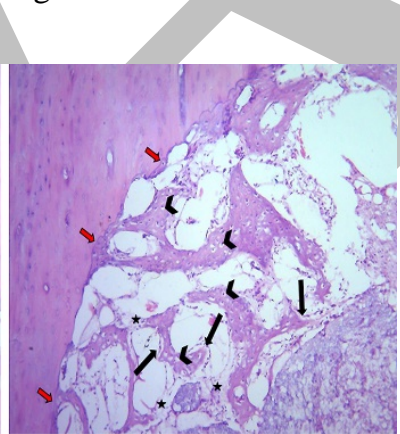

(B)
Figure 4: (A) LM of the defect site of study group (2 weeks) showing the newly formed bony spicules (arrows) extending from all the defect margins toward its center and for variable distances. The spicules of the formed bone surround the graft particles (stars). Other parts of the defect are still empty (arrow heads). (H\&Ex 40)

(B) LM of higher magnification of the previous micrograph inset showing the structure of the bony spicules which are surrounded by active osteoblasts (long arrows) and have rich osteocyte content (arrow heads). Some regions of the bone spicules are still unmineralized (stars). Line of osteointegration between the newly formed bone and the native bone can be seen (short red arrows) (H\&E $\mathrm{x} 100)$

\section{Eight weeks interval}

Histological examination of specimens obtained from group A (control) revealed more amount of bone formation in relation to the 2 weeks group. The bone appeared denser and consisted of small newly formed osteons surrounding some remnants of the graft material. Some areas of immature bone which contained numerous irregularly arranged osteocytes were seen in between the adult bone. Complete osteointegration between the old and new bone was seen (Figure 5).
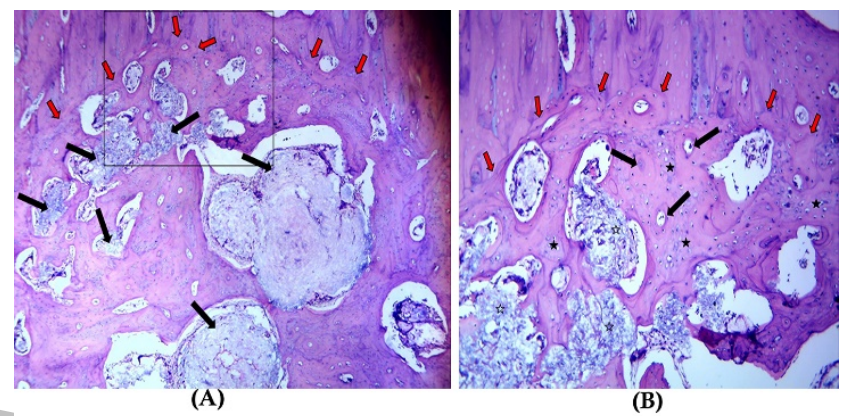

(B)

Figure 5: (A) LM of the defect site of the control group (8 weeks) showing the healing bone which occupies a large surface area of the defect space. The formed bone almost consists of dense bone which surround remnants of graft material (large black arrows). Complete osteointegration (short red arrows) can be seen between the old bone and the newly formed bone. (H\&E x 40)

(B) LM of higher magnification of the previous micrograph inset showing the structure of the formed mass of bone. Newly formed small osteons can be seen (long black arrows) surrounding less mature areas of bone (solid stars). Note the line of demarcation (short red arrows) between the new and old bone and remnants of the graft material (hollow stars). (H\&E x 100)

In group B (study), similar observations were noted like the control group but more amount of bone were seen filling most of the defect site. This new bone mass consisted mostly of compact bone enclosing small areas of immature bone (Figure 6).

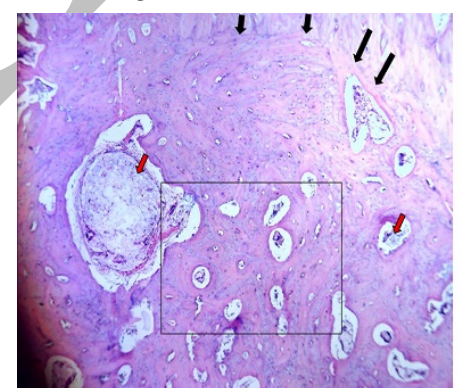

(A)

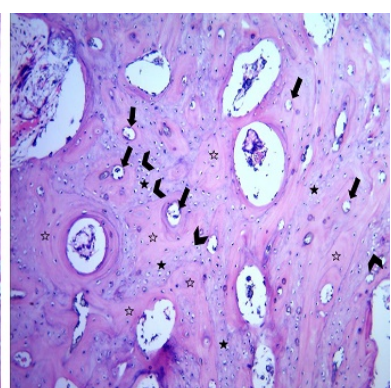

(B)
Figure 6: (A) LM of the defect site of the study group (8 weeks) showing the density of the formed bone which almost completely fill the defect area. Complete osteointegration between the native bone and the newly formed bone mass can be seen (long black arrows). Some remnants of the graft material (short red arrows) can still be seen. (H\&E x 40)

(B) LM of higher magnification of the previous micrograph inset showing the organization of the formed compact bone which consists of numerous osteons (black arrows). Note the different stainability of the formed bone with areas of mature bone (hollow stars) enclosing areas of less mature bone (solid stars). These less mature areas contain numerous irregularly distributed osteocytes lacunae (arrow heads). (H\&E x 100) 


\section{Histomorphometric results to evaluate bone density}

Table 1 showed comparison between the two studied period groups (2,8 weeks) according to the mean percentage of bone surface area in the control group (Bone graft alone). The mean percentage of bone surface area was $40.00 \%$ after 2 weeks \& $66.80 \%$ after 8 weeks, and that increase was statistically significant as p value was $\mathrm{p} 1=0.001$.

Table 1 showed comparison between the two studied period groups (2,8weeks) according to the mean percentage of bone surface area in the study group(MPM). The mean percentage of bone surface area was $47.0 \%$ after 2 weeks $\$ 74.80 \%$ after 8 weeks, and that increase was statistically significant as $\mathrm{p}$ value was $\mathrm{p} 2 \leq 0.001$.

Table (1): Comparison between the two studied periods according to mean bone percentage results in control and study groups $(\mathrm{n}=5)$.

\begin{tabular}{|c|c|c|c|c|}
\hline $\begin{array}{l}\text { Mean bone } \\
\text { percentage } \\
\text { results in } \\
\text { control } \\
\text { groups }\end{array}$ & 2 weeks & 8 weeks & $\mathbf{t}$ & $\mathbf{p}$ \\
\hline $\begin{array}{l}\text { Min. - Max. } \\
\text { Mean } \pm \text { SD. } \\
\text { Median }\end{array}$ & $\begin{array}{l}37.0-42.0 \\
40.00 \pm 1.87 \\
40\end{array}$ & $\begin{array}{l}62.0 \quad- \\
70.0 \\
66.80 \pm \\
3.11 \\
67\end{array}$ & $127.595^{*}$ & $=0.001^{*}$ \\
\hline $\begin{array}{l}\text { Sig. bet. } \\
\text { periods }\end{array}$ & $\mathrm{P}_{1}=0.001^{*}$ & & & \\
\hline $\begin{array}{l}\text { Mean bone } \\
\text { percentage } \\
\text { results in } \\
\text { study } \\
\text { groups } \\
\end{array}$ & 2 weeks & 8 weeks & $\mathbf{t}$ & p \\
\hline $\begin{array}{l}\text { Min. - Max. } \\
\text { Mean } \pm \text { SD. } \\
\text { Median }\end{array}$ & $\begin{array}{l}42.0-50.0 \\
47.0 \pm 3.16 \\
48\end{array}$ & $\begin{array}{l}71.0 \quad- \\
78.0 \\
74.80 \pm \\
3.27 \\
75\end{array}$ & $77.943^{*}$ & $<0.001^{*}$ \\
\hline $\begin{array}{l}\text { Sig. bet. } \\
\text { periods }\end{array}$ & & & & \\
\hline
\end{tabular}

t: Student t-test

$\mathrm{p}_{1}$ : $\mathrm{p}$ value for comparing between 2 weeks and 8 weeks in control groups.

$\mathrm{p}_{2}$ : p value for comparing between 2 weeks and 8 weeks in study groups

*: Statistically significant at $\mathrm{p} \leq 0.05$

Table 2 showed comparison between the control and the 2 study groups according to the mean percentage of bone surface area. There was a statistically significant difference between them at the 2 periods ( $2 \& 8$ weeks) where the $\mathrm{p}$ value was (0.001), respectively.

Table 3 showed comparison between the 2 study groups according to the change in the mean percentage of bone surface area at each study period.

There was no statistically significant difference between them as $p$ value was $(p=0.008)$.
Table (2): Comparison between the two studied groups according to mean bone percentage results.

\begin{tabular}{|l|l|l|l|l||}
\hline $\begin{array}{l}\text { Mean bone } \\
\text { percentage results }\end{array}$ & $\begin{array}{l}\text { Control } \\
(\mathbf{n = 5})\end{array}$ & $\begin{array}{l}\text { Study } \\
(\mathbf{n}=\mathbf{5})\end{array}$ & $\mathbf{t}$ & $\mathbf{p}$ \\
\hline $\mathbf{2}$ Weeks & & & & \\
Min. - Max. & $37.0-42.0$ & $42.0-50.0$ & & \\
Mean \pm SD. & $40.00 \quad \pm$ & $47.0 \pm 3.16$ & $4.260^{*}$ & $0.003^{*}$ \\
Median & 1.87 & 48 & & \\
\hline $\mathbf{8}$ Weeks & 40 & 48 & & \\
Min. - Max. & $62.0-70.0$ & $71.0-78.0$ & & \\
Mean \pm SD. & 66.80 & $\pm 74.80 \quad \pm$ & $3.961^{*}$ & $0.004^{*}$ \\
Median & 3.11 & 3.27 & & \\
\hline
\end{tabular}

t: Student t-test

$\mathrm{p}$ : p value for comparing between the two groups

*: Statistically significant at $\mathrm{p} \leq 0.05$

Table (3): Comparison between the two studied groups according to change of mean bone percentage results.

\begin{tabular}{||l|l|l|l|l||}
\hline $\begin{array}{l}\text { Change of mean } \\
\text { bone } \\
\text { percentage } \\
\text { results }\end{array}$ & Control & Study & U & $\mathbf{p}$ \\
\hline $\mathbf{2 - 8}$ Weeks & $(\mathbf{n = 5 )}$ & & \\
Min. - Max. & $22.0-25.0$ & $\begin{array}{l}23.0 \quad- \\
33.0 \\
27.80 \quad \pm\end{array}$ & 1.0 & \\
Mean \pm SD. & $23.40 \quad \pm$ & 0.008 \\
Median & 23.14 & 28 & & \\
\hline
\end{tabular}

U: Mann Whitney test

$\mathrm{p}$ : $\mathrm{p}$ value for comparing between the two groups

\section{DISCUSSION}

The hypothesis of this study was that the use of concentrated growth factors combined with a bone graft material could yield better results in terms of quantity and quality of osseous regeneration compared to the use of bone graft alone.

Limited studies are reported in the literature concerning the MPM. However, the effect of growth factors on bone healing and osseointegration has been extensively investigated. Lee et al. (27), in an animal study reported that the use of platelet enriched fibrin glue, a similar preparation to MPM, results in a statistically significant amount of bone gain. This result agreed with our study, where a new concept of fabricating growth factors-enriched bone graft matrix has been introduced, this offers the MPM the positional stability by stabilizing the bone particles, preserving its shape with subsequent "in situ" immobilization of the component of ridge preservation materials and this was the logical evolution of the fibrin glues function to incorporate platelets within the products (28).

The current study revealed the osteo-conductive potentials of both materials. In this study the newly formed bone was assessed histologically and histomorphometrically, and from the statistical analysis of the histomorphometric results, MPM showed better quality 
of bone formation regarding the homogenisity and the increased amount of the newly formed bone.

However, an interesting finding was that although the study group showed to be more significant in bone density, but the change in the mean percentage of bone was not statistically significant comparing the two groups demonstrated by $\mathrm{p}$ value $(\mathrm{p}=0.008)$. This means that the mean bone density is significantly higher in the study group but the pattern of improvement is nearly the same throughout time comparing the two groups. This could be attributed to the fact that the fibrin matrix in which the bone graft is suspended does not have any osteoconductive properties by its own, but the mineral fraction is the one that gives such property.

These findings agreed with Dr. Perissé (29) in its histological studies revealed the presence of monocytes inside the MPM biopsies, these monocytes are very important in bone formation as they allow a regulation of production of BMPs which are highly important proteins in the induction of bone production

Also, MPM is rich with growth factors EL Moheb (21) claimed that the MPM is the only natural and autogenous product that can offer stability to bone graft particles. He conducted a study and compared MPM to PRF and favored MPM biologically and histologically, which again was proved by our results but compared with bone graft instead revealing higher regenerative features of MPM.

Our study supported Choukroun et al. (11) results who reported a 30\% decrease in the healing time needed for sinus floor augmentation prior to dental implant placement when the graft material is used in combination with plasma concentrates. The authors attributed this accelerated healing to the fibrin network and platelets growth factors. This could explain the stability of the graft volume after loading in the study group compared to the control group.

Also during manipulation, the retention in the fibrous mesh of the bone fragments or the grafting material conserves its cohesion and avoids its departure away from the recipient bed.

The use of MPM in periodontology and implant therapy has a great impact in the outcome of the grafting surgery because it enhances transport of thematerial by securing its implementation as explained before by Mazzoni and Perisse (22).

Therefore from this study we found that the use of bone filler combined with fibrin, platelets and leukocytes in the form of MPM shows a better histological evidence of hard bone formation as evidenced by its high osteoblastic activity and maturation, also proved by the histomophometrical results regarding the higher $\mathrm{p}$ value in the MPM group.

\section{CONCLUSION}

Within the limitation of our study we concluded that:

1. Both MPM and Bone graft induced new bone formation, however, MPM possesses osseconductive as well as osseoinductive abilities.

2. MPM not only enhances the rate of new bone formation, but also increases the quality (density) of the newly-formed bone.

3. MPM can be very effective for reconstruction of bony defects.

4. MPM reduced dispersion of the bone graft particles which may act as anaid in reducing the amount of the needed bone graft.
5. Mineralized plasmatic matrix is a cost-effective source of growth factors and is easy to prepare.

\section{CONFLICT OF INTEREST}

The authors declare that they have no conflicts of interest.

\section{REFERENCES}

1. D'aquino R, De Rosa A, Lanza V, Tirino V, Laino L, Graziano A. Human mandible bone defect repair by the grafting of dental pulp stem/progenitor cells and collagen sponge biocomplexes. EUR Cell Mater. 2009;18:75-83.

2. Saikia K, Bhattacharya T, Bhuyan S,Talukdar D,Saikia S,Jitesh P. Calcium phosphate ceramics as bone graft substitutes in filling bone tumor defects. Indian J Orthop. 2008;42:169-72.

3. Finkemeier C. Bone grafting and bone-graft substitutes. J Bone Joint Surg Am. 2002;84:454-64.

4. Giannoudis P, Dinopoulos H, Tsiridis E. Bone substitutes: An update. Injury J. 2005;36:20-7.

5. Singh S. Management of infra bony defects in mandibular molars in a patient with generalized aggressive periodontitis using autogenous bone graft from maxillary tuberosity. J Indian SocPeriodontol. 2010;14:53-6.

6. Kim S. Bone grafting using particulate dentin. Key Eng Mater. 2007;342:29-32.

7. Gutwald R. Mesenchymal stem cells and inorganic bovine bone mineral in sinus augmentation: comparison with augmentation by autologous bone in adult sheep. Br J Oral MaxillofacSurg. 2009;6:226-9.

8. Suárez-González D, Barnhart K, Saito E, Vanderby R, Hollister S, Murphy W. Controlled nucleation of hydroxyapatite on alginate scaffolds for stem cell-based bone tissue engineering. JBiomed Mater Res A. 2010;95:222-34.

9. Dolores J, Enrique M, and Nayeli I. Platelet-Rich Plasma Peptides: Key for Regeneration. Inter J Peptid. 2012;20:10-5.

10. Lucarelli E, Beretta R, Dozza B, Tazzari P, O’Connell S, Ricci F, et al. A recently developed bifacial platelet- rich fibrin matrix.Eur Cell Mater. 2010;20:13-23.

11. Choukroun J, Diss A, Simonpieri A, Girard MO, Schoeffler C. Platelet-rich fibrin (PRF): a secondgeneration platelet concentrate. Part IV: clinical effects on tissue healing. Oral Surg Oral Med Oral Pathol Oral RadiolEndod. 2006;101:56-60.

12. Kiran N, Mukunda K, Tilak TN. PlateletConcentrates. J Dent SciRes. 2011;2:50-61.

13. Sunitha R, Munirathnam N. Platelet-rich fibrin: evolution of a second-generation platelet concentrate. Indian J Dent Res. 2008;19:42-6.

14. Ambica K, Sarika C, Sangeeta T, Mahesh V. Endodontic management of single rooted immature mandibular second molar with single canal using MTA and platelet-rich fibrin membrane barrier: A case report. J ClinExp Dent. 2011;3:487-90.

15. Dohan D, Choukroun J, Diss A, Dohan S. Platelet-rich fibrin (PRF): a second-generation platelet concentrate. Part I: technological concepts and evolution. Oral Surg Oral Med Oral Pathol Oral RadiolEndod. 2006;101:37-44.

16. Rodella LF, Favero G, Boninsegna R, Buffoli B, Labanca M, Scari G, et al. Growth factors, CD34 positive cells, and fibrin network analysis in concentrated growth factors fraction. Microsc Res Tech. 2011;74:772-7. 
17. Marx RE, Carlson ER, Eichstaedt RM, Schimmele SR, Strauss JE, Georgeff KR. Platelet-rich plasma: Growth factor enhancement for bone grafts. Oral Surg Oral Med Oral Pathol Oral RadiolEndod. 1998;85:638-46.

18. Bashkar SN. Orban' oral histology and embryology. 11th ed. St. Louis, Baltimore, Boston, Chicago, London, Philadelphia, Sydney, Toronto: Mosby; 1990. pp 365, 33941,349-50,470.

19. Werner S, Grose R. Regulation of wound healing by growth factors and cytokines. Physiol Rev. 2003;83:83570.

20.Périssé J, de Bérail A, Lauwers F, Lopez R. Interest of Mineralized Plasmatic Matrix in Secondary Autogenous Bone Graft for the Treatment of Alveolar Clefts. J Craniofac Surg. 2015;26:2148-51.

21.EL Moheb M. The use of growth factors fibrin network to enhance architecture, mechanical and biological aspect of the graft particles. Int J Pre Clin Dent Res. 2014;23:41-4.

22. Mazzoni L, Périssé J. Apports de la microscopie électronique à balayage pour la Matrice Plasmatique Minéralisée. La Lettre de la Stomatologie 2011;51:14-21.

23. Carleton HM, Drury RAB, Wallington EA. Carleton histological technique: Oxford University Press, USA;1980.

24. Eriskan EF, Axelrod DW, Melsen F. Bone histomorphometry. New York: Raven Press; 1994. p 3-12.

25. Ferreira T, Rasband W. Image J user guide. IJI 46r NatlInst Health, Bethesda, MD 2012.

26. Kirkpatric LA, Feeney BC.A simple guide to IBM SPSS: for version 20.0: Nelson Education; 2012.

27. Lee HJ, Choi BH, Jung JH, Zhu SJ, Lee SH, Huh JY,et al. Maxillary sinus floor augmentation using autogenous bone grafts and platelet-enriched fibrin glue with simultaneous implant placement. Oral Surg Oral Med Oral Pathol Oral RadiolEndod. 2007;103:329-33.

28. DohanEhrenfest DM, Bielecki T, Jimbo R, Barbé G, Del Corso M, Inchingolo F,et al. Do the fibrin architecture and leukocyte content influence the growth factor release of platelet concentrates? An evidence-based answer comparing a pure platelet-rich plasma (P-PRP) gel and a leukocyte-and platelet-rich fibrin (L-PRF). Curr Pharm Biotechnol. 2012;13:1145-52.

29. Perisse J, Alami HB, Vassalaky LM, Caveriviere P, Betito M, Marchou M. Aspect clinique et histologique des Matrices Plasmatiques Minéralisées (MPM). Revue d'implantologie. 2012:23-5. 